this quotient group. This implies that this quotient group is abelian and of type $(2,2)$ when $p=2$, and when $p>2$ it must contain at least $p$ invariant cyclic subgroups of order $p^{2}$. As this is contrary to the fact that $G$ contains $p+1$ conjugate sets which involve generating operators of its maximal cyclic subgroups, we have proved that we arrive at an absurdity by assuming that $G$ does not involve any operator of order $p^{m-1}$, $m>3$.

When $p>2$ there are only two non-cyclic groups of order $p^{m}$ which involve operators of order $p^{m-1}, m>3$, and each of these clearly contains maximal cyclic subgroups of order $p^{a}$ which are transformed into themselves by more than $p^{a+1}$ operators of $G$. Hence it results that the three non-cyclic groups of order $2^{m}$ which were considered above in the second paragraph are the only non-cyclic groups of order $p^{m}$ in which every maximal cyclic subgroup is transformed into itself by at most $p$ times as many operators of the group as there are operators in this maximal subgroup. This completes the proof of the theorem in question, and hence we can assume that every non-cyclic group of order $p^{m}$, with the exception of the three of order $2^{m}$ which involve one and only one cyclic subgroup of order $2^{m-1}$, contains at least one maximal cyclic subgroup of order $p^{\alpha}$ which is transformed into itself by more than $p^{\alpha+1}$ operators of the group.

UNIVERSITY OF ILLINOIS.

\title{
AN EXPRESSION FOR THE GENERAL TERM OF A RECURRING SERIES.
}

BY PROFESSOR TSURUICHI HAYASHI.

Professor Arthur Ranum has given in the Bulletin, volume 17, No. 9, June, 1911, pages 457-461, an explicit form of the general term of a recurring series rationally in terms of the first few terms and the constants of the scale of relation. I will give here another more explicit and more convenient form without demonstration.

Let $u_{0}+u_{1}+u_{2}+\ldots+u_{n}+\ldots$ be any recurring series of order $n$, and let

$$
u_{m}=a_{1} u_{m-1}+a_{2} u_{m-2}+\cdots+a_{n} u_{m-n} \quad(m \geqq n)
$$


be its scale of relation. Then for any value of $m$ not less than $n$,

where

$$
u_{m}=\sum_{\nu=0}^{\nu=n-1} u_{\nu} A_{\nu}
$$

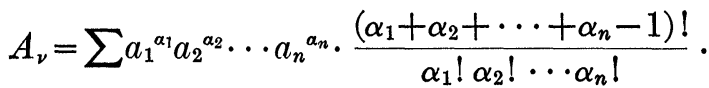

$$
\begin{aligned}
& \left(\alpha_{n}+\alpha_{n-1}+\cdots+\alpha_{n-\nu}\right),
\end{aligned}
$$

the summation extending over all the positive integral and zero values of $\alpha_{1}, \alpha_{2}, \cdots, \alpha_{n}$, for which

$$
\alpha_{1}+2 \alpha_{2}+3 \alpha_{3}+\cdots+n \alpha_{n}=m-\nu .
$$

Since the formula is true when $m=n$, and assuming it to be true for the values $n+l, n+l-1, \cdots, n+1, n$ of $m$, we can prove it by mathematical induction to be true for the next value $n+l+1$, without much difficulty, unless $l<n$. Even for the case $l<n$, a slight attention leads us to the same result.

More generally, it may also be shown that for any value of $m$ greater than $\lambda$

where

$$
u_{m}=\sum_{\nu=\lambda-n+1}^{\nu=\lambda} u_{\nu} A_{\nu}
$$

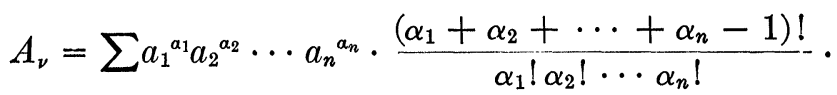

$$
\begin{aligned}
& \left(\alpha_{n}+\alpha_{n-1}+\cdots+\alpha_{\lambda-\nu+1}\right) \text {, }
\end{aligned}
$$

the summation extending over all the positive integral and zero values of $\alpha_{1}, \alpha_{2}, \cdots, \alpha_{n}$ for which

$$
\alpha_{1}+2 \alpha_{2}+3 \alpha_{3}+\cdots+n \alpha_{n}=m-\nu .
$$

TôHOKU IMPERIAL UNIVERSITY,

SendaI, Japan,

July, 191. 